\title{
Correlative Structural, Biochemical and Behavioral Study in Neuroprotection with Palmitoylehanolamide after Perinatal Asphyxia in the Immature Brain
}

\author{
María Inés Herrera ${ }^{1}{ }^{2}$, Tamara Kobiec ${ }^{1}$ Lucas Daniel Udovin ${ }^{2}{ }^{*}$, Nicolás Toro-Urrego ${ }^{2}$, Carlos Federico \\ Kusnier $^{2}$, Juan Pablo Luaces ${ }^{2}$, Rodolfo Kolliker ${ }^{2}$ and Francisco Capani ${ }^{2}{ }^{*}$ \\ 1. Centro de Investigaciones en Psicología y Psicopedagogía, Facultad de Psicología, Universidad \\ Católica Argentina, Buenos Aires, Argentina. \\ 2. Instituto de Investigaciones Cardiológicas (ININCA), UBA-CONICET, CABA, Buenos Aires, \\ Argentina. \\ 3. Departamento de Biología Facultad de Farmacia y Bioquímica, UAJFK. \\ * Corresponding author: franciscocapani@hotmail.com
}

Perinatal asphyxia (PA) is an obstetric complication associated with an impaired gas exchange. Diminish in the oxygen levels prompted short and long-term alterations in synapses and related structures that are related to neuronal dysfunction and death. This serious health problem could lead to neonatal mortality and continues to be a determinant of several neurodevelopmental disorders. The lack of knowledge about the proper neuroprotective agents prompted us to investigate the role of Palmitoylethanolamide (PEA) in the experimental treatment of PA. This natural occurring amide has exerted protective effects in several models of brain injury and neurodegeneration.

For this study, we used a well-established murine model of PA [1]. After one, of moderate PA (19 min) one group of rats were sacrificed and their brains were analyzed by combining immunohistochemistry, conventional electron microscopy, and 3-D reconstruction. The other group was subjected to behavioural analysis [2].

After one month of the PA insult, we observed an augmentation in CA1 neuronal nucleus degeneration using electron microscopy (Fig 1). Immunohistochemistry revealed a significant increase for $\mathrm{pNF} H / \mathrm{M}$ and decrease in MAP-2 in CA1 reactive area. These changes were also observed when analyzing the level of expression of these markers by western blot. Vertical exploration impairments and anxietyrelated behaviors were encountered in the OF and EPM tests. PEA treatment attenuated PA-induced hippocampal damage and its corresponding behavioral alterations.

These results contribute to the elucidation of PEA neuroprotective role after PA and the future establishment of therapeutic strategies for the developing brain.

References:

[1] F Capani et al., Exp Neurol. 219 (2019), p. 404.

[2] MI Herrera et al., Front Neurosci. 12 (2018), p. 145. doi: 10.3389/fnins.2018.00145. 

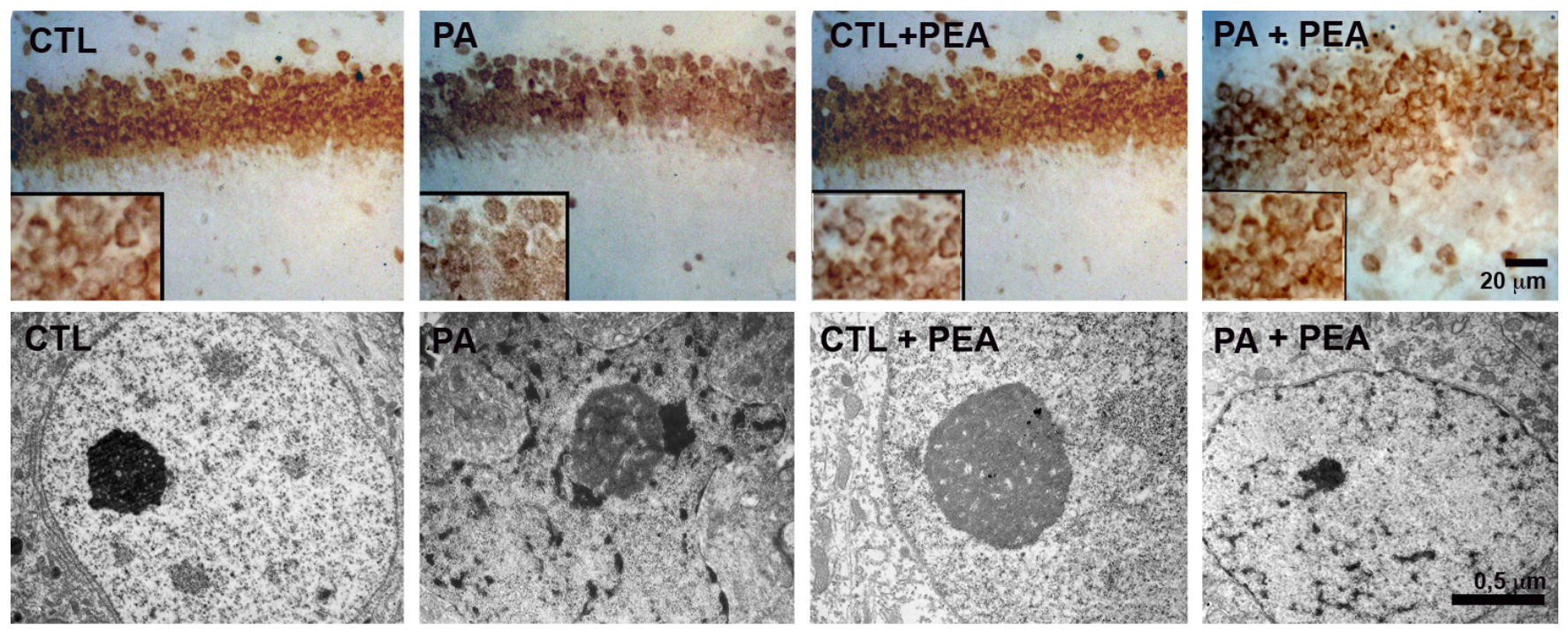

Figure 1. Neuronal alterations induced by PA and recovery after PEA treatment. (A-D) Micrographs of Stratum radiatum of CA1 hippocampal area in the different groups analyzed by NeuN Immunostaining. $(\mathrm{E}-\mathrm{H})$. Electron microscopy (EM) of CA1 neurons showing that most of the condensed cells correspond to neurons in degeneration. Statistical analysis of different parameters was performed. Significant differences were calculated by two-way analysis of variances (ANOVAs) tests followed by post hoc comparisons using Student's t-test (two-tailed) adjusted by Bonferroni correction. 